doi:10.1136/thoraxjnl-2012-202678.337

'U Griesenbach, ${ }^{2} \mathrm{M}$ Inoue, ' $\mathrm{C}$ Meng, ${ }^{1} \mathrm{R}$ Farley, ${ }^{1} \mathrm{M}$ Chan, 'NK Newman, ${ }^{1} \mathrm{~A}$ Brum, 2J You, ${ }^{3} \mathrm{~A}$ Kerton, ${ }^{4} \mathrm{~A}$ Shoemark, ${ }^{5} \mathrm{AC}$ Boyd, ${ }^{1} \mathrm{JC}$ Davies, ${ }^{1} \mathrm{TE}$ Higgins, ${ }^{6} \mathrm{DR}$ Gill, ${ }^{6} \mathrm{SC}$ Hyde, ${ }^{5} \mathrm{JA}$ Innes, ${ }^{5} \mathrm{DJ}$ Porteous, ${ }^{2} \mathrm{M}$ Hasegawa, ${ }^{1}$ EWFW Alton. 'Department of Gene Therapy, Imperial College London, London, UK; ${ }^{2}$ DNAVEC Corporation, Tsukuba, Japan; ${ }^{3}$ Central Biomedical Services, Imperial College London, London, UK; ${ }^{4}$ Paediatric Department, Royal Brompton Hospital, London, UK; ${ }^{M}$ Medical Genetics Section, Centre for Molecular Medicine, MRC Institute of Genetics and Molecular Medicine University of Edinburgh, Western General Hospital, Edinburgh, UK; ${ }^{6}$ Gene Medicine Group, Nuffield Dept of Clinical Laboratory Sciences, University of Oxford, John Radcliffe Hospital, Oxford, UK

Rational Our ongoing efforts to improve pulmonary gene transfer thereby enabling gene therapy for the treatment of lung diseases such as cystic fibrosis (CF) has led to the development of a lentiviral vector (SIV) pseudotyped with the Sendai virus envelope proteins $\mathrm{F}$ and $\mathrm{HN}$.

Objectives Here, we begin to place this vector onto a translational pathway to the clinic, by addressing some key milestones that have to be achieved.

Main results These include: (1) a single dose produces lung expression for the life-time of the mouse (approximately 2 years), (2) only brief contact time is needed to achieve transduction, (3) repeated daily administration leads to a dose-related increase in gene expression, (4) repeated monthly administration to mouse lower airways is feasible without loss of gene expression, (5) there is no evidence of chronic toxicity during a 2 year study period, (6) F/HNSIV transduction generates persistent gene expression in human differentiated airway cultures, and human lung slices and transduces freshly obtained primary human airway epithelial cells.

Conclusions The data support F/HN-pseudotyped SIV as a promising vector for pulmonary gene therapy for a number of diseases including CF and we are now undertaking the necessary refinements to progress this vector into clinical trials.

\section{P96 REPEAT ADMINISTRATION OF GL67A/PGM169 IS FEASIBLE, SAFE, AND PRODUCES ENDOGENOUS LEVELS OF CFTR EXPRESSION AFTER 12 DOSES}

doi:10.1136/thoraxjnl-2012-202678.338

'EWFW Alton, ${ }^{2} \mathrm{AC}$ Boyd, ${ }^{3} \mathrm{SH}$ Cheng, ${ }^{1} \mathrm{~J}$ Davies, ${ }^{4} \mathrm{LA}$ Davies, ${ }^{5} \mathrm{~A}$ Dayan, ${ }^{4} \mathrm{DR}$ Gill, ${ }^{1} \mathrm{U}$ Griesenbach, ${ }^{1} \mathrm{~T}$ Higgins, ${ }^{4} \mathrm{SC}$ Hyde, ${ }^{2} \mathrm{~A}$ Innes, ${ }^{6} \mathrm{G}$ McLachlan, ${ }^{2} \mathrm{D}$ Porteous, ${ }^{4} \mathrm{I} A$ Pringle, ${ }^{3}$ RK Scheule, ${ }^{4} \mathrm{SG}$ Sumner-Jones. 'Department of Gene Therapy, Imperial College London, London, UK; ${ }^{2}$ Medical Genetics Section, Molecular Medicine Centre, University of Edinburgh, Edinburgh, UK; ${ }^{3}$ Genzyme, a Sanofi Company, Framingham, MA, USA ${ }^{4}$ Gene Medicine Group, NDCLS, Oxford University, Oxford, UK; ${ }^{5}$ Toxicology Consultant, London, UK; ${ }^{6}$ Roslin Institute, University of Edinburgh, Edinburgh, UK

For gene therapy to improve lung function in CF subjects, repeated administration of the gene transfer agent over the lifetime of a patient will be necessary. This requirement limits the usefulness of adenoviral and adeno-associated viral vectors (both commonly used in CF gene therapy in the past), because these viral vectors induce adaptive immune responses which render repeat dosing ineffective. Thus, for CF gene therapy non-viral vectors are currently the only viable option. We have, therefore, undertaken an extensive preclinical research programme to select the most efficient non-viral vector for a Multi-dose CF trial. The cationic lipid formulation GL67A was ultimately chosen and combined with a CpG-free CFTR plasmid (pGM169). We recently completed a single-dose Phase $2 \mathrm{a}$ clinical safety and molecular efficacy study, which demonstrated proof-of principle for long lasting (several weeks) correction of the CF-specific chloride transport defect, but also indicated that the toxicity-efficacy window is comparatively narrow. To support the trial, we undertook a murine repeat dose (12 doses over
6 months) efficacy, biodistribution and toxicology study. We show that (a) repeated lung administration of pGM169/GL67A is safe and feasible using a clinically relevant nebuliser, (b) achieves reproducible, dose-dependent and persistent gene expression (>140 days after each dose) and importantly (c) allows for a cumulative treatment effect (as measured by levels of vector-specific mRNA) particularly when working in a clinically relevant dosing range (dose 1: $0.8 \%$ vector-specific/endogenous mCftr (range 0-1.8), dose 12: $74 \%$ vector-specific/endogenous mCftr (range 31.6-204). The results of this repeat dosing study in the mouse therefore (1) demonstrate that endogenous levels of CFTR mRNA can be achieved by exogenously applied gene therapy (cumulative effect), and (2) affirm the UK CF Gene Therapy Consortium's strategy of repeat dosing.

\section{P97 TRANSITION FROM A DISTRICT GENERAL HOSPITAL (DGH) CLINIC TO A DISTANT ADULT CENTRE}

doi:10.1136/thoraxinl-2012-202678.339

SL Prudon, R Casans, F Hampton. James Cook University Hospital, Middlesbrough, United kingdom

Background Transition between CF centres in the same town is well reported but there is less work on transition from large DGH clinics to distant adult centres. Our local paediatric unit provides total care for 60 patients. The majority will move on to the adult centre in a city 45 miles away. Until the last 2 years transition had been taking place very late because low staffing at the adult unit limited outreach support for the local patients who were thus reluctant to move on. The paediatric team felt that although transition was hampered by the lack of a local adult centre an increase in adult staffing was an opportunity to review current practiscs and identify areas for improvement.

Aims To identify ways in which the transition process could be improved and to explore the need for the introduction of formal transition clinics.

Methods Adolescents (13-18 years) within the paediatric clinic and young adults (18-26 years) who had completed transition to the adult centre in the last two years were surveyed using a proforma merging the standards of the CF trust and Department of Health. The CF nurses also completed questionnaires about these patients.

Results 16/19 identified patients took part. CF nurse results showed that only $37 \%$ of patients started transition between ages 13 and 16 and only 50\% had a key worker. Whilst the nurses felt that they offered most teenagers the opportunity to be seen alone only $14 \%$ of patients stated they were seen on their own and most said they would strongly prefer it. Discussions of careers, finance, higher education and fertility took place in up to $43 \%$, but commonly after age 16 . Patients reported wanting more information on these topics. Although $60 \%$ of patients marked the adult clinic was easy to reach and had appropriate facilities, the 'free comments' section revealed difficulties.

Conclusions There are areas of practise that can be improved in the paediatric clinic independently of the setting up of transition clinics. More teenagers need to be seen on their own and cheque lists are needed to ensure all relevant areas are discussed.

\section{P98 CF TRAINING IN THE UK}

doi:10.1136/thoraxjnl-2012-202678.340

D Nazareth, M Walshaw. BTS CF Specialist Advisory Group, London, United Kingdom

Background As survival in CF improves and the adult population continues to grow, there is an increasing need for the adequate training of chest physicians to care for this patient group. To reflect this, in 2010 the UK respiratory specialist training 
curriculum was strengthened, setting out guidance for the minimum requirements for CF training to achieve CCT. However, because the curriculum is already full we wished to assess whether the current training schemes in the UK can cope with this increased need. We have already canvassed the views of the trainees ${ }^{1}$, and now wished to assess the views of those tasked with organising this training.

Method We surveyed all 19 UK Training Program Directors (Adult Respiratory Medicine), asking about availability of CF experience and how this new guidance was being applied for their 596 trainees.

Results Only 12 Directors (63\%) were aware of trainees with a special interest in CF (26, half currently gaining out-of-programme experience [OOPE]). Northern Ireland (NI) and SE Scotland had most trainees pursuing an interest in CF ( $22 \%$ and $20 \%$ respectively), where $75 \%$ of these were undertaking OOPE.

Only 1 centre (NI) had changed trainee allocation arrangements to accommodate the 2010 curriculum changes, but despite this trainees rotated to a specialist CF centre in only 12 Deaneries (63\%), where the average training time was 3 months. About 180 trainees $(30 \%)$ did not rotate to a specialist centre, and in these cases Directors reported that individuals were required to make their own arrangements $(26 \%)$ or had organised day-release or training days $(18 \%)$.

Conclusions This survey highlights that, despite the increasing numbers of adult CF patients and the need for suitable training for our future respiratory colleagues now reflected in the curriculum, a significant proportion of trainees in the UK still have limited exposure to CF during their training. Further representations have been made to the training authorities to reinforce the need for increased CF training.

Reference:

1. CF Experience for Respiratory Trainees in the UK. Journal of Cystic Fibrosis 2012,11 (Suppl1), S127, 274

\section{Pulmonary rehabilitation}

\section{P99 PULMONARY REHABILITATION IN INTERSTITIAL LUNG DISEASE PATIENTS: EFFECTS ON MAXIMUM EXERCISE CAPACITY, ANXIETY AND DEPRESSION}

doi:10.1136/thoraxjnl-2012-202678.341

${ }^{1} \mathrm{MM}$ Peasey, ${ }^{2} \mathrm{SS}$ Kon, 'D Dilaver, ${ }^{2} \mathrm{JL}$ Canavan, 'MG Ng, ${ }^{2} \mathrm{SE}$ Jones, 'AL Clark, ${ }^{2} \mathrm{Ml}$ Polkey, ${ }^{2}$ WD-C Man. ${ }^{1}$ Harefield Pulmonary Rehabilitation Team, Royal Brompton and Harefield NHS Foundation Trust, Harefield, Middlesex, United Kingdom; '2Respiratory Biomedical Research Unit, Royal Brompton and Harefield NHS Foundation Trust, Harefield, Middlesex, United Kingdom

Background There is increasing evidence to support the use of Pulmonary Rehabilitation (PR) in patients with Interstitial Lung Disease (ILD). Several studies have shown significant improvements in six minute walk distance and health related quality of life measures, such as the Chronic Respiratory Disease Questionnaire (CRQ), following PR (Holland et al; 2008). However there is a paucity of data surrounding other outcome measures such as maximal walking exercise tests (the incremental shuttle walk: ISW) or anxiety and depression levels. As the ISW and the Hospital Anxiety and Depression scale (HAD) are commonly used outcome measures in UK PR programmes, the aim of the study was to assess the response of these outcomes to PR in the ILD population.

Methods We analysed outcome data in 62 (30 male:32 female) consecutive ILD patients completing an 8-week outpatient PR programme. Diagnoses were idiopathic pulmonary fibrosis $(n=29)$, connective tissue related ILD $(n=8)$, sarcoidosis $(n=14)$, hypersensitivity pneumonitis $(n=3)$, asbestosis $(n=4)$, drug induced ILD $(n=4)$. Pre- and post-PR data was analysed using either Paired T-Tests or Wilcoxon Tests.

Results Baseline mean (standard deviation) age was 70(11) years, FVC $69(22) \%$ predicted and median $\left(25^{\text {th }}, 75^{\text {th }}\right.$ centiles) MRC dyspnoea score was $4(3,4)$. There was a significant improvement in ISW (95\% confidence intervals 31, 69 metres), HAD-Anxiety $(95 \% \mathrm{CI}-0.8,-2.4)$ and HAD-Depression $(95 \% \mathrm{CI}$ $-0.7,-2.3)$. There were also significant improvements in all domains of the CRO.

Conclusion The ISW and HAD scores are responsive to PR in patients with ILD.

\section{P100 THE CLINICAL COPD QUESTIONNAIRE: RESPONSE TO PULMONARY REHABILITATION}

doi:10.1136/thoraxjnl-2012-202678.342

'D Dilaver, 'MM Peasey, 'AL Clark, 'MGS Ng, ${ }^{2} \mathrm{M}$ Mittal, ${ }^{2}$ SSC Kon, ${ }^{2} \mathrm{JL}$ Canavan, ${ }^{2}$ SE Jones, ${ }^{2} \mathrm{MI}$ Polkey, ${ }^{2}$ WDC Man. ${ }^{1}$ Harefield Pulmonary Rehabilitation Team, Harefield, Middlesex, United Kingdom; ${ }^{2}$ Respiratory Biomedical Research Unit, Royal Brompton \& Harefield NHS Foundation Trust, Harefield, Middlesex, United Kingdom

Background The Clinical COPD Questionnaire (CCQ) is a 10-item health status instrument which has been shown to be reliable and valid in COPD. It takes only two minutes to complete and is simple to score, ranging from 0 (best) -6 (worst health status). A change in the total CCQ score of 0.4 or more is considered clinically significant (Kocks et al Respir Res 2006). There is a relative paucity of data assessing the responsiveness of the CCQ to pulmonary rehabilitation (PR). We hypothesised that the CCQ would be responsive to PR and that changes would correlate with changes in other well established health status instruments (Chronic Respiratory Questionnaire: CRQ, St George's Respiratory Questionnaire: SGRQ and the COPD Assessment Test: CAT).

Methods 75 consecutive COPD patients referred to an 8-week outpatient PR programme were recruited. The CCQ, along with the CRQ, SGRO, CAT, and incremental shuttle walk (ISW), were measured before and after PR. Paired t-test was used to compare outcomes before and after PR, whilst Spearman's rank correlation was used to assess association between change in CCO with change in other health status questionnaires.

Results 53 patients completed PR. Baseline characteristics were 33 Male:20 Female, mean (standard deviation) age 68.5(9.9) years, FEV1\% predicted 58 (27) and ISW 224 (178) metres. There was a significant reduction (improvement) in CCQ following PR (Pre: 2.9 (1.3) versus Post: 2.1 (1.2); 95\% confidence interval -0.4 to $-1.0)$. Significant improvements were also seen in ISW, CRQ domains, SGRQ and CAT with PR. Changes in CCQ correlated significantly with changes in the other health status instruments (see table 1).

Conclusion The CCO is responsive to $\mathrm{PR}$ and a practical alternative to longer-established health status instruments.

Abstract P100 Table 1 Relationship between change in CCO and change in CRO, SGRO and CAT with PR $\Delta=$ Change in PR

\begin{tabular}{lcc}
\hline$\Delta$ CCO & Rho & p-value \\
\hline$\Delta$ CRO Dyspnoea & -0.44 & 0.001 \\
$\Delta$ CRO Fatigue & -0.44 & 0.001 \\
$\Delta$ CRO Emotion & -0.34 & 0.01 \\
$\Delta$ CRQ Mastery & -0.46 & $<0.001$ \\
$\Delta$ SGRQ Symptoms & 0.42 & 0.005 \\
$\Delta$ SGRQ Activities & 0.59 & $<0.001$ \\
$\Delta$ SGRQ Impact & 0.60 & $<0.001$ \\
$\Delta$ SGRQ Total & 0.65 & $<0.001$ \\
$\Delta$ CAT & 0.64 & $<0.001$ \\
\hline
\end{tabular}

